\title{
Gastrointestinal toxicity during induction treatment for childhood acute lymphoblastic leukemia: The impact of the gut microbiota
}

De Pietri, Silvia; Ingham, Anna Cäcilia; Frandsen, Thomas L.; Rathe, Mathias; Krych, Lukasz; Castro Mejía, Josue L.; Nielsen, Dennis S.; Nersting, Jacob; Wehner, Peder S.; Schmiegelow, Kjeld Total number of authors: 13

Published in:

International Journal of Cancer

Link to article, DOI:

10.1002/ijc.32942

Publication date:

2020

Document Version

Peer reviewed version

Link back to DTU Orbit

\section{Citation (APA):}

De Pietri, S., Ingham, A. C., Frandsen, T. L., Rathe, M., Krych, L., CastroMejía, J. L., Nielsen, D. S., Nersting, J., Wehner, P. S., Schmiegelow, K., Hasle, H., Pamp, S. J., \& Müller, K. (2020). Gastrointestinal toxicity during induction treatment for childhood acute lymphoblastic leukemia: The impact of the gut microbiota. International Journal of Cancer, 147(7), 1953-1962. https://doi.org/10.1002/ijc.32942

\section{General rights}

Copyright and moral rights for the publications made accessible in the public portal are retained by the authors and/or other copyright owners and it is a condition of accessing publications that users recognise and abide by the legal requirements associated with these rights.

- Users may download and print one copy of any publication from the public portal for the purpose of private study or research.

- You may not further distribute the material or use it for any profit-making activity or commercial gain

- You may freely distribute the URL identifying the publication in the public portal 


\section{Gastrointestinal toxicity during induction treatment for childhood acute lymphoblastic leukemia: the impact of the gut microbiota.}

Silvia De Pietri ${ }^{1}$, Anna Cäcilia Ingham ${ }^{2,3}$, Thomas Leth Frandsen ${ }^{1}$, Mathias Rathe ${ }^{4,5}$, Lukasz Krych $^{6}$, Josue Leonardo Castro Mejia ${ }^{6}$, Dennis Sandris Nielsen ${ }^{6}$, Jacob Nersting ${ }^{1}$, Peder Skov Wehner $^{4}$, Kjeld Schmiegelow ${ }^{1,7}$, Henrik Hasle ${ }^{8}$, Sünje Johanna Pamp² ${ }^{2}$ Klaus Müller ${ }^{1,7,9}$.

${ }^{1}$ Department of Paediatrics and Adolescent Medicine, Rigshospitalet, Copenhagen University Hospital, Copenhagen, Denmark.

${ }^{2}$ Research Group for Genomic Epidemiology, Technical University of Denmark, Kongens Lyngby, Denmark.

${ }^{3}$ Department of Bacteria, Parasites and Fungi, Statens Serum Institut, Copenhagen, Denmark.

${ }^{4}$ Hans Christian Andersen Children’s Hospital, Odense University Hospital, Odense, Denmark.

${ }^{5}$ OPEN, Odense Patient Data Explorative Network, Odense University Hospital, Odense, Denmark.

${ }^{6}$ Department of Food Science, University of Copenhagen, Copenhagen, Denmark

${ }^{7}$ Institute of Clinical Medicine, Faculty of Medicine, University of Copenhagen.

${ }^{8}$ Department of Paediatrics, Aarhus University Hospital, Aarhus, Denmark.

Institute for Inflammation Research, Rigshospitalet, Copenhagen University Hospital, Copenhagen, Denmark.

Corresponding author: Silvia De Pietri, MD, PhD, The Paediatric Oncology Research Laboratory, Department of Paediatrics and Adolescent Medicine, Copenhagen University Hospital,

This article has been accepted for publication and undergone full peer review but has not been through the copyediting, typesetting, pagination and proofreading process which may lead to differences between this version and the Version of Record. Please cite this article as doi: 10.1002/ijc.32942 
Rigshospitalet, Blegdamsvej 9, 2100 Copenhagen, Denmark. Phone number: +45 35459828. Email: silvia.de.pietri@regionh.dk; depietri.silvia@gmail.com.

Short title: chemotherapy-induced gastrointestinal toxicity and gut microbiota.

Key words: gastrointestinal toxicity, mucositis, acute lymphoblastic leukemia, microbiota, citrulline, C-reactive protein.

Article category: research article.

\section{List of abbreviations:}

IM, intestinal mucositis

ALL, acute lymphoblastic leukemia

SR, standard risk

IR, intermediate risk

HR, high risk

NOPHO, Nordic Society of Pediatric Hematology and Oncology

CRP, C-reactive protein

spp., species

HSCT, hematopoietic stem cell transplantation 
RH, Rigshospitalet, University Hospital of Copenhagen

OUH, H.C. Andersen Children’s Hospital, Odense University Hospital

AUH, Aarhus University Hospital, Skejby

NCI-CTCAE, National Cancer Institute Common Terminology Criteria for Adverse Events

p.o., oral

i.v., intravenous

ASV, amplicon sequence variant

db-RDA, distance-based redundancy analyses

IQR, interquartile range

FDR, false discovery rate

AML, acute myeloid leukemia

GVHD, graft-versus-host disease -

\section{Ivovelty and Impact:}

This is the first study investigating the associations between the gut microbiota and the severity of chemotherapy-induced intestinal mucositis in a group of homogeneously treated children with acute lymphoblastic leukemia. Severe enterocyte loss and systemic inflammation were associated with loss of microbial alpha diversity, loss of Lachnospiraceae species and overgrowth of enterococci. 
Maintaining a diverse microbial community rich in short-chain fatty acids-producing bacteria, such as Lachnospiraceae, may be important to protect against chemotherapy-induced intestinal damage.

Abstract: 241 words

Body of the text: 4339 words

Figures: 4

Tables: 2

Supplemental tables and figures: 9

References: 50

\section{Abstract:}

Intestinal mucositis is a common side effect of chemotherapy leading to diarrhea, abdominal pain and increased risk of infections. The intestinal microbiota has been recognized as a key regulator of mucosal immune responses. Therefore, we hypothesized that intestinal microbial changes would be associated with enterocyte loss and systemic inflammation during induction treatment for childhood acute lymphoblastic leukemia (ALL). We prospectively included 51 children newly-diagnosed with ALL treated in Denmark in 2015-2018. Plasma C-reactive protein (CRP), plasma citrulline (marker of functional enterocytes mass) measurements and fecal samplings were performed on treatment days 1, 8, 15, 22 and 29. Moreover, intestinal mucositis was scored by a trained nurse/physician. Fecal samples in patients and 19 healthy siblings were analyzed by 16S rRNA gene sequencing (V3-V4 region). Bacterial alpha diversity was lower in patients compared to siblings. It decreased 
from day 1 to days 8-22 and increased on day 29. Shannon alpha diversity index was correlated with CRP on days 15-29 (rho=-0.33--0.49; P<0.05) and with citrulline on days 15 and 29 (although with P-values $<0.06$, rho=0.32-0.34). The abundance of unclassified Enterococcus species (spp.) was correlated with CRP on days 22-29 (rho=0.42-0.49; $\mathrm{P}<0.009$ ), while the abundance of unclassified Lachnospiraceae spp. was correlated with citrulline on days 8-15 (rho=0.48-0.62, $\mathrm{P}<0.001$ ). Systemic inflammation, enterocyte loss and relative abundance of unclassified Enterococcus spp. reached a peak around day 15. In conclusion, specific changes in the microbiota were associated with the severity of enterocyte loss and systemic inflammation during chemotherapy. 


\section{Introduction:}

The overall survival of childhood acute lymphoblastic leukemia (ALL) has dramatically improved during the last two decades ${ }^{1}$. However, several patients still experience serious acute toxicities. Intestinal mucositis (IM) is one of the most common side effects of chemotherapy, resulting from disruption of the immunological balance of the intestinal mucosal barrier ${ }^{2}$. It can present with severe abdominal pain, vomiting and diarrhea, leading to increased opioid consumption and risk of malnutrition $^{3,4}$. Besides suffering from debilitating symptoms, patients with IM are at increased risk of systemic infections as enhanced mucosal permeability facilitates bacterial translocation into the blood stream ${ }^{5}$. Moreover, products from intestinal bacteria and damaged cells released during mucositis stimulate a local and systemic inflammatory response, leading to fever ${ }^{2,5,6}$. This systemic inflammation may be involved in the pathogenesis of other chemotherapy-induced organ toxicities by inducing endothelial activation and circulatory disturbances. This mechanism has mainly been investigated in hematopoietic stem cell transplantation (HSCT) complications ${ }^{7}$. However, it may be at play also for toxicities occurring during ALL treatment, such as hepatic sinusoidal obstruction syndrome, pancreatitis and thromboembolism. Therefore, understanding the factors influencing IM verity and the related systemic inflammation is crucial to identify new preventive measures and reduce the overall toxic burden of chemotherapy. IM mucositis severity can be objectively estimated by measuring plasma citrulline level. Citrulline is an amino acid produced almost exclusively by enterocytes and its circulating levels are considered a reliable marker of the functional enterocytes mass ${ }^{8}$. 
Several lines of evidence suggest that the intestinal microbiota may be involved in different steps of the IM pathogenesis ${ }^{9}$. Commensal bacteria are thought to increase the strength of the mucosal barrier by stimulating tight junction proteins expression, mucus production and enterocyte metabolism ${ }^{10,11}$. Moreover, commensal bacteria have a crucial role in regulating mucosal inflammatory responses ${ }^{12}$. Therefore, alteration of the host-microbiota interactions during chemotherapy may influence the degree of tissue damage and inflammatory response. Several preclinical and clinical studies have described changes in the intestinal microbiota during chemotherapy ${ }^{13,14}$. Nevertheless, the role of these changes in influencing the severity of IM remains unknown. Such understanding may lead to new preventive strategies targeting the intestinal microbiota.

The aim of this prospective study was to describe changes in the intestinal microbiota during induction treatment for childhood ALL, and investigate its associations with enterocyte loss, systemic inflammation and bacteremia.

\section{Method:}

Study population:

Fifty-one children (1-18 years old) newly-diagnosed with ALL and treated according to the Nordic Society of Paediatric Haematology and Oncology (NOPHO) ALL 2008 protocol were prospectively included at Rigshospitalet, University Hospital of Copenhagen (RH), H.C. Andersen Children's Hospital, Odense University Hospital (OUH), and Aarhus University Hospital, Skejby (AUH), Denmark, between December 2015 and March 2018. Patients received a 29-days induction treatment as previously described ${ }^{15}$. Within nine weeks from treatment initiation, one healthy 
sibling aged 1-18 years was included for each patient (with priority to the one closest in age). Out of 51 families, 36 were screened for siblings' eligibility whereof 12 refused participation, four did not meet the inclusion criteria, and one was excluded due to missing samples. Therefore, 19 siblings were included. All siblings shared the same parents and housing as the patients.

Intestinal mucositis, systemic inflammation and bacteremia

IM severity was scored on treatment days 1 (baseline), 8, 15, 22 and 29 by a trained physician or study nurse by summation of the scores for abdominal pain and diarrhea according to the National Cancer Institute Common Terminology Criteria for Adverse Events (NCI-CTCAE) 4.0 criteria, as follows: grade 0 (0-1 points), grade I ( $2-3$ points) and grade II ( $\geq 4$ points). Plasma citrulline was measured as a marker of enterocyte loss in patients on treatment days 1, 8, 15, 22, 29 and in ten unrelated healthy controls (five male and five female blood donors aged 21-32 years). Plasma was isolated from EDTA-anticoagulated blood samples by centrifugation at 1000xg for 10 min and stored at $-80^{\circ} \mathrm{C}$ within two hours. Citrulline was measured using a Waters Acquity ${ }^{\mathrm{TM}}$ UltraPerformance Liquid Chromatography system with a Tandem Quadrupole detector ${ }^{16}$. Plasma Creactive protein (CRP) was measured on treatment days 8, 15, 22 and 29 by automated Immunoturbidimetric assay, as a marker of systemic inflammation (upper normal limit $10 \mathrm{mg} / \mathrm{L}$ ). Positive blood cultures and antibiotic administrations during induction treatment were registered from the patients’ medical records. Bacteremia episodes were defined as one or more positive blood cultures for bacteria or fungi taken less than one week apart. All patients received Pneumocystis pneumonia prophylaxis with sulphamethoxazole and trimethoprim at 20 and $4 \mathrm{mg} / \mathrm{kg} / \mathrm{day}$, respectively, twice weekly (which were not registered). 


\section{Fecal samples collection}

In patients, fecal samples were collected on treatment days 1 (range -2-5), 8 (range 5-11), 15 (range 13-18), 22 (range 20-25) and 29 (range 27-34), for a total of 185 samples (median 4, range $\vdash 5$, samples per patient). In siblings, fecal samples were collected at baseline (2-62 days from the patients' start of chemotherapy) and after six months (22-29 weeks after the baseline sample), for a total of 30 samples (11 siblings with samples both at baseline and at six-months follow up). Siblings did not receive any antibiotics during the month prior to fecal sampling. Samples were stored at $20^{\circ} \mathrm{C}$ immediately after collection and then transferred to $-80^{\circ} \mathrm{C}$ within a few days.

\section{DNA extraction and $16 S$ rRNA gene amplicon sequencing}

DNA was extracted from fecal samples and 11 blank controls using the Qiagen ${ }^{\circledR}$ QIAamp Fast DNA Stool mini kit, modified to include a bead-beating step (Qiagen ${ }^{\circledR}$ PowerBead Tubes, Garnet $0.70 \mathrm{~mm}$ ). High-throughput amplicon sequencing of the V3-V4 region of the 16S rRNA gene was conducted by Illumina ${ }^{\circledR}$ MiSeq technology (Illumina Inc., San Diego, CA, USA) at the Multi Assay Core facility (DMAC), Technical University of Denmark. Full-length sequence of the forward primer: nxt_341F: 5'TCGTCGGCAGCGTCAGATGTGTATAAGAGACAGCCTACGGGNGGCWGCAG and the reverse primer: nxt_805R: GTCTCGTGGGCTCGGAGATGTGTATAAGAGACAGGACTACHVGGGTATCTAATCC ${ }^{17}$. Samples were re-sequenced if the read count after chimera removal (described below) was $<9000$. 16S rRNA gene data processing: 
Primer sequences were removed with cutadapt (version 1.16$)^{18}$ at a tolerated maximum error rate of 15\%. Reads were further processed using the R package DADA2 (version 1.8) for inference of high-resolution amplicon sequence variants $(\mathrm{ASV})^{19}$. Forward and reverse reads were truncated at 280 bp and 200 bp, respectively, to allow overlap for merging. Chimeras were identified samplewise and removed from the whole data set (removeBimeraDenovo() function, method “consensus”). Taxonomy was assigned with SILVA reference database version 132 formatted for DADA2 ${ }^{20}$. No technical batch effects by sequencing run, extraction round, extraction kit or extraction date were detected by Principal Coordinates Analysis with different distance metrics (Bray-Curtis, Jaccard, Raup-Crick, Chao and Horn-Morisita) using the R package phyloseq. A total of 266 putative contaminant ASVs were removed using the R package decontam, following the "combined" method with probability threshold $0.07^{21}$ and manually assessing borderline sequences. Subsequently, only ASVs with at least five reads in at least two samples were selected for further analyses by using the $\mathrm{kOverA}($ ) function in the $\mathrm{R}$ package genefilter. After this step, median sequencing depth was 20649 reads (range 5038-326811) in patients' samples and 15080 reads (range 5509-302273) in siblings’ samples.

account for the variation in samples' sequencing depth and reduce the number of null counts, rarefaction to 5000 reads per sample was performed using QIIME2 ${ }^{22}$, and ASVs with overall abundance $<0.1 \%$ were excluded. Feature tables were summarized at the species level for further analyses. After this step, median sequencing depth was 5000 (range 4981-5000) in patients' samples and 5000 (range 4994-5000) in siblings' samples. Bacterial alpha diversity indicating within-sample microbial diversity was calculated at the species level as observed number of species and Shannon index (accounting for both abundance and evenness of the species present) using 
QIIME2 based on the raw ASV table by subsampling to 5000 reads/sample iterated ten times. For each sample, the mean alpha diversity parameters of the ten repetitions were used for further statistical analyses.

\section{Statistical analyses:}

The Chi-squared test was used to compare the sex distribution between patients and siblings, and the Mann-Whitney U test was used to compare continuous variables between two independent groups (age and alpha diversity in patients vs. siblings, citrulline level in patients vs. controls, citrulline and CRP levels in patients with vs. without IM, and days of antibiotics in patients with vs. without bacteremia). Wilcoxon signed-rank test was used for comparison of alpha diversity in siblings between baseline and six-months follow-up and comparison of relative abundance between baseline and later time points. Changes over time in IM severity were investigated by generalized linear mixed model with cumulative log-link for ordinal outcomes with a random intercept by patient. Analysis of response profile with unstructured variance-covariance matrix was used to investigate changes over time in citrulline level, log-transformed CRP level, log-transformed observed species and Shannon index, and to test differences in alpha diversity in patients with vs. without bacteremia and with vs. without antibiotic exposure (i.v. or p.o.) during induction treatment. Interaction between time-point and antibiotic exposure was tested by likelihood-ratio test. Statistical significance at each time-point and between groups was based on Wald test. Spearman's rank correlation test was used to investigate same-day associations between citrulline and CRP, citrulline and alpha diversity, and CRP and alpha diversity on days 8-29, as well as associations between early alpha diversity measures (day 1 and day 8) and CRP and citrulline 
measurements on day 15. Logistic regression analysis was used to investigate the association between early alpha diversity (day 1 and day 8) and bacteremia during the rest of induction treatment including only patients who completed induction treatment.

Distance-based redundancy analyses (db-RDA) based on Bray-Curtis distance matrices were used to test associations between the overall microbial community structure at the species level and plasma CRP and citrulline levels measured on the same days during chemotherapy. The analyses were conducted on CRP and citrulline separately by analyzing samples from days 8-29, regardless of time. The models were considered significant with permutational test $\mathrm{P}<0.05$. The association of the most discriminatory bacterial species according to the models (e.g. those with the top-10 scores in absolute importance over the canonical axis) with CRP or citrulline were then tested: their abundances (read counts) were correlated with CRP or citrulline levels by Spearman's rank correlation tests with false discovery rate (FDR) correction of the p-values. Species that were found significantly correlated with CRP or citrulline using samples from days 8-29 together were further tested for correlations with CRP and citrulline levels on each day separately by Spearman's rank correlation tests (without FDR-correction of the p-values). The same approach was used to restigate if specific species on day 1 or 8 were associated with later outcomes including: the occurrence of bacteremia after day 1 or day 8 (excluding patients with bacteremia before day nine) both including and excluding patients who did not take any antibiotics during induction treatment, CRP on day 15, and citrulline on day 15 . P-values $<0.05$ were considered statistically significant. Statistical analyses were conducted using the statistical software R version 3.5.1.

Ethics: 
The project was approved by the Scientific Ethics committee of the Capital Region of Denmark (H16016942) and the Danish Data Protection Agency (RH-2016-214). Parents/legal guardians of patients and siblings gave oral and written informed consent for participation in the study. No informed consent was required for the use of plasma samples from anonymous blood donors. Part of this cohort has been previously published ${ }^{23}$.

\section{Data availability:}

The 16S rRNA gene sequences are available through the European Nucleotide Archive (ENA) at the European Bioinformatics Institute (EBI) under accession number PRJEB35526.

\section{Results:}

A total of 51 children newly diagnosed with ALL were included in the study (Table 1). The 19 siblings had similar sex distribution compared to patients $(9 / 19,47 \%$, vs $38 / 51,75 \%$, males, respectively, $\mathrm{P}=0.14$ ) but were significantly older at inclusion (median years 6.8 , interquartile range [IQR] 4.6-9.6 vs. 3.7, IQR 2.7-6.8, P=0.02).

\section{Gastrointestinal toxicity and systemic inflammation}

niter day 1, 27/50 (54\%) patients presented with IM at least once during induction treatment: 21 (42\%) of grade I and 6 (12\%) of grade II. From day 1, IM severity increased reaching a peak on day 15, and then decreased on day 22 and 29 while remaining significantly higher compared to baseline (Figure 1A, Table S1). Median citrulline level in patients on day 1 was not significantly different compared to healthy controls (23.4 $\mu \mathrm{M}$, IQR 21.6-26.6, $\mathrm{P}=0.07, \mathrm{~N}=10$ ). Median citrulline level decreased during chemotherapy reaching a nadir on day 8-15 (Figure 1B). From day 8, median 
plasma CRP level increased reaching a peak on day 15 and then decreased on days 22 and 29 (Figure 1C). Plasma citrulline and CRP levels were inversely correlated on day 15 (rho=-0.61, $\mathrm{P}<0.001$ ), day 22 (rho=-0.35, $\mathrm{P}=0.016$ ), and day 29 (rho=-0.41, $\mathrm{P}=0.004)$. Patients with clinical IM (grade $\geq$ I) had lower median citrulline level than patients without IM on day 15 (12.95 $\mu \mathrm{M}$, IQR 8.33-15.23 vs. $15.85 \mu \mathrm{M}$, IQR 12.31-18.62, $\mathrm{P}=0.03)$ and day $22(15.10 \mu \mathrm{M}, \mathrm{IQR}$ 10.95-18.23 vs. 19.90 $\mu \mathrm{M}$, IQR 17.25-24.07, P=0.005). Patients with clinical IM had higher median CRP level than patients without IM on day 15 (21 mg/L, IQR 8-104 vs. $3.5 \mathrm{mg} / \mathrm{L}, \mathrm{IQR} 1-18.25, \mathrm{P}=0.002)$ and day 22 (9.5 mg/L, IQR 1.9-31.5 vs. $1 \mathrm{mg} / \mathrm{L}, \mathrm{IQR} 1-2.0, \mathrm{P}=0.014)$. The frequencies of valid IM scorings, citrulline and CRP measurements for each time-point are reported in Table S2.

\section{Bacteremia and antibiotic exposure}

Out of 49 patients completing induction treatment, 13 (27\%) experienced bacteremia with first positive blood culture on median day 13 (range 5-20). One patient had two bacteremia episodes. Out of 14 episodes, 9 (64\%) were caused by Gram-positive bacteria (together with fungi in one case) while 5 (36\%) were caused by Gram-negative bacteria (Table S3). Out of 51 patients, 45 (88\%) received antibiotics during induction treatment: 34/51 (67\%) received only i.v. antibiotics lile 11/51 (22\%) received also one p.o. agent (amoxicillin/clavulanic acid or vancomycin or ciprofloxacin or metronidazole). Of the 45 patients receiving i.v. antibiotics, 9 (20\%) received i.v. monotherapy with meropenem $(n=8)$ or piperacillin/tazobactam $(n=1)$ while $36(80 \%)$ received combined i.v. therapy with 2-7 agents (Table S4). Antibiotics (p.o. or i.v.) were started on median day 1 (range 1-14) and terminated on median day 24 (range 7-35), for a median duration of 18 days (range 4-34) of therapy. Patients experiencing bacteremia had longer antibiotic exposure 
compared to patients without bacteremia (median 23, range 9-34, vs. median 13, range 0-32 days, $\mathrm{P}=0.006)$.

\section{Alpha diversity during induction treatment}

At baseline, alpha diversity was lower in patients compared to siblings (median number of observed species: 94, IQR 65-134, vs. 135, IQR 113-174, P=0.05; median Shannon index: 4.5, IQR 3.9-5.3, vs. 5.7, IQR 5.1-6.2, P=0.002, Figure S1). Similar results were obtained when including only baseline patients’ samples taken before/on treatment day $1(\mathrm{n}=14)$.

Alpha diversity decreased from day 1 to days 8-22, and then increased again on day 29 (Figure 2). Patients who did not receive any antibiotics (6/51, 12\%) had overall higher alpha diversity during induction treatment compared to patients receiving antibiotics (observed species $\mathrm{P}<0.001$; Shannon index $\mathrm{P}=0.029$, Figure S2), with no significant interaction between antibiotic exposure and timepoint. For the 45 patients receiving antibiotics, changes in alpha diversity over time were similar to the ones observed in the overall cohort (Figure S2). In siblings, alpha diversity did not change from baseline to six-months follow up ( $\mathrm{P}>0.7)$. The number of fecal samples available for each timepoint is reported in Table S2.

\section{Alpha diversity and CRP, citrulline and bacteremia}

Shannon diversity index and plasma CRP levels were negatively correlated on days 15, 22 and 29 (Figure 3A). The same pattern was seen for the observed number of species, although the correlation was statistically significant only on day 22 (day 15: rho=-0.34, P=0.054; day 22: rho=0.40, $\mathrm{P}=0.013$; day 29: $\mathrm{rho}=-0.27, \mathrm{P}=0.090$ ). In line with this, high Shannon index tended to be associated with high plasma citrulline on days 15 and 29 (Figure 3B). Observed number of species 
and plasma citrulline were not significantly correlated on any of the days. On day 22, patients who experienced bacteremia during induction treatment had lower Shannon index compared to patients without bacteremia ( $\mathrm{P}=0.02$, Figure $\mathrm{S} 3$ ), also after excluding from the analysis patients who did not receive any i.v. or p.o. antibiotics, while the observed number of species did not differ between these two patient groups at any time-point. Alpha diversity metrics on day 1 and day 8 were not associated with subsequent CRP and citrulline levels or the occurrence of bacteremia during the rest of induction treatment.

\section{Bacterial species associated with CRP and citrulline levels}

When investigating the association between bacterial community structure and same-day plasma CRP levels by db-RDA including days 8-29 regardless of time, two taxa among the most discriminatory species were found significantly correlated with CRP. The abundance of unclassified Enterococcus species (spp.) was positively correlated with CRP level (Spearman's rho=0.32, noncorrected and FDR-corrected $\mathrm{P}<0.001$ ) while the abundance of Sutterella wadsworthensis was negatively correlated with CRP level (Spearman's rho=-0.23, non-corrected $\mathrm{P}=0.005$, FDRcorrected $\mathrm{P}=0.02)$. Correlations between unclassified Enterococcus spp., Sutterella wadsworthensis

d CRP were subsequently investigated on each day separately (Table 2).

When investigating the association between bacterial community structure and same-day plasma citrulline level by db-RDA including days 8-29 regardless of time, a positive correlation was found between the abundance of unclassified Lachnospiraceae spp. and citrulline (Spearman's rho=0.44, non-corrected and FDR-corrected P <0.001). Correlations between unclassified Lachnospiraceae spp. and citrulline subsequently investigated on each day separately were significant on days 8 and 15 (Table 2). 
Due to the findings of an association between unclassified Enterococcus spp., Sutterella wadsworthensis and plasma CRP, we specifically investigated the correlations between these taxa and plasma citrulline on each day by Spearman's rank correlation tests. The abundance of unclassified Enterococcus spp. was negatively correlated with citrulline level on day 29 (rho=-0.35, $\mathrm{P}=0.030$ ), while Sutterella wadsworthensis abundance was positively correlated with citrulline level on day 15 (rho=0.37, $\mathrm{P}=0.040$ ). Due to the findings of an association between unclassified Lachnospiraceae spp. and plasma citrulline, we specifically tested the correlations between this taxon and plasma CRP on each day by Spearman's rank correlation tests. The abundance of unclassified Lachnospiraceae spp. was negatively correlated with CRP level on day 15 (rho=-0.37, $\mathrm{P}=0.037)$.

The median relative abundance of unclassified Enterococcus spp. increased after day 1 reaching a peak on day 15 and subsequently decreased (Figure 4A). On the contrary, the median relative abundance of unclassified Lachnospiraceae spp. decreased from day 1 to day 15 and subsequently increased, however not significantly (Figure 4B).

The bacterial community structure on day 1 or day 8 was not associated with later outcomes including bacteremia during the rest of induction treatment, and CRP and citrulline levels on day 15. The 50 most abundant species during induction treatment in relation to CRP and citrulline levels are illustrated as heatmaps in Figures S4 and S5.

\section{Discussion:}

This is the first study describing weekly changes of the intestinal microbiota during induction treatment for childhood ALL and its associations with markers of intestinal toxicity and systemic 
inflammation. Bacterial alpha diversity was lower in patients compared to siblings at baseline. Alpha diversity further decreased after one week of therapy remaining significantly reduced until day 22. Low alpha diversity was associated with low plasma citrulline and high CRP levels during the period of maximum intestinal mucositis. High abundance of unclassified Lachnospiraceae spp. was associated with high citrulline and low CRP levels while high abundance of unclassified Enterococcus spp. was associated with high CRP and low citrulline levels.

Previous studies have observed reduced bacterial alpha diversity and altered intestinal microbial composition in ALL patients at diagnosis compared to controls regardless of antibiotics use ${ }^{24,25}$. Leukemia-induced dysbiosis and reduced intestinal epithelial integrity have been observed in mice models for acute myeloid leukemia (AML), with microbial changes facilitating leukemia progression ${ }^{26}$. Although alpha diversity in our patients may have been influenced by antibiotics administered before hospital admission and diagnosis, which were not registered in the hospital records, these findings encourage further investigations of a possible role of intestinal dysbiosis in ALL pathogenesis and progression.

The observed rapid decrease in microbial diversity after chemotherapy initiation is in line with previous studies in patients undergoing $\operatorname{HSCT}^{13,27-29}$ and chemotherapy for AML and ALL ${ }^{30,31}$. The observed changes in microbial diversity paralleled IM symptoms, enterocyte loss and systemic inflammation, suggesting an association between low microbial diversity and IM. The increase in alpha diversity at the end of the four-week induction treatment may also have reflected the more intensive use of antibiotics during the first three weeks. However, reduced microbial diversity was associated with increased enterocyte loss and systemic inflammation, supporting the hypothesis that 
dysbiosis may influence IM severity. Accordingly, studies in mice have demonstrated reduced microbial diversity during chemotherapy-induced $\mathrm{IM}^{32,33}$, with low diversity being associated with increased intestinal permeability ${ }^{32}$, reduced villus length and crypt depth, and increased proinflammatory cytokines in the small intestine ${ }^{33}$. In the clinical setting, reduced alpha diversity has been associated with radiotherapy-induced diarrhea ${ }^{34}$ and other intestinal inflammatory diseases such as acute graft-versus-host disease $(\mathrm{GVHD})^{28,35}$ and inflammatory bowel diseases ${ }^{36}$. Nevertheless, clinical studies have mainly focused on describing chemotherapy-induced dysbiosis rather than directly addressing the association between microbial composition and IM severity.

In this study, high abundance of unclassified Lachnospiraceae spp. was associated with increased citrulline and reduced CRP levels around the time-point of maximum IM, suggesting a potential protective role of these bacteria against intestinal barrier injury and systemic inflammation. Contrarily to our expectations, the relative abundance of unclassified Lachnospiraceae spp did not vary significantly over time, although it was lowest at the time-point of maximum IM severity and inflammation. Lachnospiraceae are known producers of butyrate, a short-chain fatty acid derived from fermentation of dietary fiber ${ }^{37}$. Besides being an important nutrient for enterocytes, butyrate eliorates epithelial barrier function by increasing the expression of mucins and tight junction proteins $^{37}$. Moreover, it reduces intestinal inflammatory responses by inhibiting the production of pro-inflammatory cytokines ${ }^{37}$. In mice receiving 5-fluorouracil, the administration of butyrate reduced intestinal barrier injury and inflammation ${ }^{38}$. Therefore, supplementation with dietary fibers, butyrate or probiotics may help limit intestinal mucosal injury during chemotherapy. Sutterella wadworthensis was associated with reduced CRP and increased citrulline. Despite commonly found in the human gut, the role of this bacteria in human disease is controversial. A recent study has 
hypothesized that it may primarily exert a commensal role, participating in maintaining immune physiological balance in the gut through its mildly pro-inflammatory properties ${ }^{39}$.

In our study, the maximum relative abundance of unclassified Enterococcus spp. coincided with the time-point of maximum IM severity and inflammation. Moreover, high abundance of unclassified Enterococcus spp. was associated with increased CRP and reduced citrulline, indicating that high abundance of enterococci may characterize patients with severe IM. Overgrowth of enterococci during chemotherapy may reflect the loss of enterocytes and Paneth cells, which produce REGIII $\gamma$, an antimicrobial peptide limiting enterococci growth ${ }^{40}$. Moreover, since REGIII $\gamma$ production is stimulated by Gram-negative bacteria, broad-spectrum antibiotics may also promote enterococci overgrowth by indirectly inhibiting REGIII $\gamma$ production ${ }^{41}$. Besides increasing during chemo- and antimicrobial therapy, as seen in patients undergoing HSCT $^{13,27,28}$ and children with acute leukemia ${ }^{42,43}$, enterococci may exacerbate intestinal inflammation by compromising gut epithelial integrity ${ }^{44}$ and stimulating macrophage activation ${ }^{45}$. Accordingly, increased enterococci abundance has been associated with acute $\mathrm{GVHD}^{28}$ and ulcerative colitis (correlating also with disease severity) ${ }^{46}$. However, further studies are needed to discriminate if terococcal overgrowth is just accompanying or promoting intestinal injury. We did not find any association between early microbial diversity or structure and later incidence of bacteremia, enterocyte loss or systemic inflammation. In children with ALL, Hakim et al. found that the microbial composition, but not alpha diversity, at the beginning of a treatment course was predictive of febrile neutropenia and diarrhea ${ }^{31}$. Predictive relationships between microbial diversity and composition and subsequent bacteremia have been demonstrated in other studies in children with cancer $^{47}$ and patients undergoing $\mathrm{HSCT}^{27,48}$. Finally, the predictive role of microbiota in the 
development of IM has mainly been demonstrated in patients receiving pelvic radiotherapy ${ }^{49}$. However, prophylaxis with probiotics has been shown to reduce the severity of chemo/radiotherapy-induced IM in several studies ${ }^{9,50}$, supporting the hypothesis that the microbial composition may constitute a predisposing factor for mucositis.

The weekly prospective sampling in this study allowed a detailed exploration of temporal patterns, and associations between microbiota, intestinal toxicity and inflammation. Nevertheless, the study is limited by the small sample size and unmeasured confounders for the microbiota. Comparisons of the microbiota between patients and siblings were limited by the size and characteristics of the sibling group (which differed in age and sex distribution from to the patient group). Moreover, the small sample size may have prevented us from detecting significant changes over time in Lachnospiraceae spp. relative abundance. Given the high prevalence and variability of antibiotics exposure, we could not accurately differentiate the effects of antibiotics and chemotherapy on the microbiota. Further, diet and the use of nasal-tube feeding may have influenced the microbiota. Finally, given the observational nature of the study, we can only demonstrate association and not causality.

In conclusion, our data suggest that maintaining a diverse gut microbiota rich in butyrate-producing bacteria, such as Lachnospiraceae, may reduce intestinal mucositis, while loss of bacterial alpha diversity and overgrowth of enterococci may exacerbate intestinal injury and inflammatory responses during chemotherapy. In future studies, investigating the associations between microbial functions and markers of gastrointestinal toxicity may help identify possible target metabolic pathways to preserve intestinal barrier integrity during chemotherapy. 


\section{Conflicts of interest}

The authors declare no conflicts of interest.

\section{Acknowledgments}

We sincerely thank The Danish Childhood Cancer Foundation, the Copenhagen University Hospital, Rigshospitalet, the A.P. Møller foundation, the Dagmar Marshall foundation, and the Knud og Edith Eriksen memorial foundation for supporting the project. Sequence pre-processing was performed using the DeiC National Life Science Supercomputer at the Technical University of Denmark.

\section{Authors contributions}

SDP, SJP and KM conceived and designed the study. SDP, TF, MR, HH, PSW and KS were involved in patients' inclusion and acquisition of samples and clinical data. JN performed the citrulline analysis. SDP performed the DNA extraction from fecal samples. SDP, ACI, and SJP contributed to 16S rRNA gene data processing. SDP, LK, JLKM, SJP, and KM contributed to the statistical analysis. KS is Principal Investigator of the NOPHO ALL2008 protocol. SDP and KM wrote the first draft of the manuscript. All authors participated in on-going data interpretation and manuscript revisions. 


\section{References:}

1. Pui C-H, Yang JJ, Hunger SP, Pieters R, Schrappe M, Biondi A, Vora A, Baruchel A, Silverman LB, Schmiegelow K, Escherich G, Horibe K, et al. Childhood Acute Lymphoblastic Leukemia: Progress Through Collaboration. J Clin Oncol 2015;33:2938-49.

2. Sonis ST. The pathobiology of mucositis. Nat Rev Cancer 2004;4:277-84.

3. Lalla R V., Bowen J, Barasch A, Elting L, Epstein J, Keefe DM, McGuire DB, Migliorati C, Nicolatou-Galitis O, Peterson DE, Raber-Durlacher JE, Sonis ST, et al. MASCC/ISOO clinical practice guidelines for the management of mucositis secondary to cancer therapy. Cancer 2014;120:1453-61.

4. De Pietri S, Nielsen BN, Ifversen M, Kielsen K, Müller KG. Morphine consumption is associated with systemic inflammation in children undergoing allogeneic hematopoietic stem cell transplantation. Immunopharmacol Immunotoxicol 2019;0:1-7.

5. van der Velden WJFM, Herbers AHE, Netea MG, Blijlevens NM a. Mucosal barrier injury, fever and infection in neutropenic patients with cancer: introducing the paradigm febrile mucositis. Br J Haematol 2014;167:441-52.

6. Kornblit B, Müller K. Sensing danger: toll-like receptors and outcome in allogeneic hematopoietic stem cell transplantation. Bone Marrow Transplant 2017;52:499-505.

7. Schots R, Kaufman L, Riet I Van, Othman T Ben, Waele M De, Camp B Van, Demanet C. Proinflammatory cytokines and their role in the development of major transplant-related complications in the early phase after allogeneic bone marrow transplantation. Leukemia 
2003;17:1150-6.

8. Fragkos KC, Forbes A. Citrulline as a marker of intestinal function and absorption in clinical settings: A systematic review and meta-analysis. United Eur Gastroenterol J 2018;6:181-91.

9. Touchefeu Y, Montassier E, Nieman K, Gastinne T, Potel G, Bruley des Varannes S, Le Vacon F, de La Cochetière MF. Systematic review: the role of the gut microbiota in chemotherapy- or radiation-induced gastrointestinal mucositis - current evidence and potential clinical applications. Aliment Pharmacol Ther 2014;40:409-21.

10. Qin H-L, Shen T-Y, Gao Z-G, Fan X-B, Hang X-M, Jiang Y-Q, Zhang H-Z. Effect of lactobacillus on the gut microflora and barrier function of the rats with abdominal infection. World J Gastroenterol 2005;11:2591-6.

11. Hamer HM, Jonkers D, Venema K, Vanhoutvin S, Troost FJ, Brummer R-J. Review article: the role of butyrate on colonic function. Aliment Pharmacol Ther 2008;27:104-19.

12. Rakoff-Nahoum S, Paglino J, Eslami-Varzaneh F, Edberg S, Medzhitov R. Recognition of Commensal Microflora by Toll-Like Receptors Is Required for Intestinal Homeostasis. Cell 2004;118:229-41.

13. Montassier E, Gastinne T, Vangay P, Al-Ghalith G a., Bruley des Varannes S, Massart S, Moreau P, Potel G, de La Cochetière MF, Batard E, Knights D. Chemotherapy-driven dysbiosis in the intestinal microbiome. Aliment Pharmacol Ther 2015;42:515-28.

14. Lin XB, Dieleman LA, Ketabi A, Bibova I, Sawyer MB, Xue H, Field CJ, Baracos VE, Gänzle MG. Irinotecan (CPT-11) Chemotherapy Alters Intestinal Microbiota in Tumour 
Bearing Rats. PLoS One 2012;7:e39764.

15. Toft N, Birgens H, Abrahamsson J, Bernell P, Griškevičius L, Hallböök H, Heyman M, Holm MS, Hulegårdh E, Klausen TW, Marquart H V, Jónsson OG, et al. Risk group assignment differs for children and adults 1-45 yr with acute lymphoblastic leukemia treated by the NOPHO ALL-2008 protocol. Eur J Haematol 2013;90:404-12.

16. Chaimbault P, Petritis K, Elfakir C, Dreux M. Determination of 20 underivatized proteinic amino acids by ion-pairing chromatography and pneumatically assisted electrospray mass spectrometry. J Chromatogr A 1999;855:191-202.

17. Illumina ${ }^{\circledR}$. 16S Metagenomic Sequencing Library Preparation. Part \#15044223 Rev B. https://support.illumina.com/documents/documentation/chemistry_documentation/16s/16smetagenomic-library-prep-guide-15044223-b.pdf. Illumina.com2013;1-28.

18. Martin M. Cutadapt removes adapter sequences from high-throughput sequencing reads. EMBnet.journal 2011;17.

19. Callahan BJ, McMurdie PJ, Rosen MJ, Han AW, Johnson AJA, Holmes SP. DADA2: Highresolution sample inference from Illumina amplicon data. Nat Methods 2016;13:581-3.

20. Callahan B. Silva taxonomic training data formatted for DADA2 (Silva version 132). Zenodo 2018;

21. Davis NM, Proctor DM, Holmes SP, Relman DA, Callahan BJ. Simple statistical identification and removal of contaminant sequences in marker-gene and metagenomics data. Microbiome 2018;6:226. 
22. Caporaso JG, Kuczynski J, Stombaugh J, Bittinger K, Bushman FD, Costello EK, Fierer N, Peña AG, Goodrich JK, Gordon JI, Huttley GA, Kelley ST, et al. QIIME allows analysis of high-throughput community sequencing data. Nat Methods 2010;7:335-6.

23. Rathe M, De Pietri S, Wehner PS, Frandsen TL, Grell K, Schmiegelow K, Sangild PT, Husby S, Müller K. Bovine Colostrum Against Chemotherapy-Induced Gastrointestinal Toxicity in Children With Acute Lymphoblastic Leukemia: A Randomized, Double-Blind, Placebo-Controlled Trial. J Parenter Enter Nutr 2019;00.

24. Rajagopala S V, Yooseph S, Harkins DM, Moncera KJ, Zabokrtsky KB, Torralba MG, Tovchigrechko A, Highlander SK, Pieper R, Sender L, Nelson KE. Gastrointestinal microbial populations can distinguish pediatric and adolescent Acute Lymphoblastic Leukemia (ALL) at the time of disease diagnosis. BMC Genomics 2016;17:635.

25. Bai L, Zhou P, Li D, Ju X. Changes in the gastrointestinal microbiota of children with acute lymphoblastic leukaemia and its association with antibiotics in the short term. $J$ Med Microbiol 2017;66:1297-307.

Ye H, Adane B, Khan N, Alexeev E, Nusbacher N, Minhajuddin M, Stevens BM, Winters AC, Lin X, Ashton JM, Purev E, Xing L, et al. Subversion of Systemic Glucose Metabolism as a Mechanism to Support the Growth of Leukemia Cells. Cancer Cell 2018;34:659-673.e6.

27. Taur Y, Xavier JB, Lipuma L, Ubeda C, Goldberg J, Gobourne A, Lee YJ, Dubin KA, Socci ND, Viale A, Perales M-A, Jenq RR, et al. Intestinal Domination and the Risk of Bacteremia in Patients Undergoing Allogeneic Hematopoietic Stem Cell Transplantation. Clin Infect Dis 
2012;55:905-14.

28. Holler E, Butzhammer P, Schmid K, Hundsrucker C, Koestler J, Peter K, Zhu W, Sporrer D, Hehlgans T, Kreutz M, Holler B, Wolff D, et al. Metagenomic Analysis of the Stool Microbiome in Patients Receiving Allogeneic Stem Cell Transplantation: Loss of Diversity Is Associated with Use of Systemic Antibiotics and More Pronounced in Gastrointestinal Graft-versus-Host Disease. Biol Blood Marrow Transplant 2014;20:640-5.

29. Ingham AC, Kielsen K, Cilieborg MS, Lund O, Holmes S, Aarestrup FM, Müller KG, Pamp SJ. Specific gut microbiome members are associated with distinct immune markers in pediatric allogeneic hematopoietic stem cell transplantation. Microbiome 2019;7:131.

30. Galloway-Peña JR, Smith DP, Sahasrabhojane P, Ajami NJ, Wadsworth WD, Daver NG, Chemaly RF, Marsh L, Ghantoji SS, Pemmaraju N, Garcia-Manero G, Rezvani K, et al. The role of the gastrointestinal microbiome in infectious complications during induction chemotherapy for acute myeloid leukemia. Cancer 2016;122:2186-96.

31. Hakim H, Dallas R, Wolf J, Tang L, Schultz-Cherry S, Darling V, Johnson C, Karlsson EA, Chang T-C, Jeha S, Pui C-H, Sun Y, et al. Gut Microbiome Composition Predicts Infection Risk During Chemotherapy in Children With Acute Lymphoblastic Leukemia. Clin Infect Dis 2018;67:541-8.

32. Forsgård RA, Marrachelli VG, Korpela · Katri, Frias R, Collado MC, Korpela R, Monleon D, Spillmann T, Österlund P. Chemotherapy-induced gastrointestinal toxicity is associated with changes in serum and urine metabolome and fecal microbiota in male Sprague-Dawley 
rats. Cancer Chemother Pharmacol 2017;80:317-32.

33. Wu C-H, Ko J-L, Liao J-M, Huang S-S, Lin M-Y, Lee L-H, Chang L-Y, Ou C-C. Dmethionine alleviates cisplatin-induced mucositis by restoring the gut microbiota structure and improving intestinal inflammation. Ther Adv Med Oncol 2019;11:175883591882102.

34. Wang A, Ling Z, Yang Z, Kiela PR, Wang T, Wang C, Cao L, Shen M, Ran X, Su Y, Cheng T, Wang J, et al. Gut Microbial Dysbiosis May Predict Diarrhea and Fatigue in Patients Undergoing Pelvic Cancer Radiotherapy: A Pilot Study. PLoS One 2015;10:e0126312.

35. Jenq RR, Ubeda C, Taur Y, Menezes CC, Khanin R, Dudakov JA, Liu C, West ML, Singer N V., Equinda MJ, Gobourne A, Lipuma L, et al. Regulation of intestinal inflammation by microbiota following allogeneic bone marrow transplantation. J Exp Med 2012;209:903-11.

36. Kolho KL, Korpela K, Jaakkola T, Pichai MVA, Zoetendal EG, Salonen A, De Vos WM. Fecal microbiota in pediatric inflammatory bowel disease and its relation to inflammation. Am J Gastroenterol 2015;110:921-30.

37. Bach Knudsen K, Lærke H, Hedemann M, Nielsen T, Ingerslev A, Gundelund Nielsen D, Theil P, Purup S, Hald S, Schioldan A, Marco M, Gregersen S, et al. Impact of DietModulated Butyrate Production on Intestinal Barrier Function and Inflammation. Nutrients 2018;10:1499.

38. Ferreira TM, Leonel AJ, Melo MA, Santos RRG, Cara DC, Cardoso VN, Correia MITD, Alvarez-Leite JI. Oral supplementation of butyrate reduces mucositis and intestinal permeability associated with 5-fluorouracil administration. Lipids 2012;47:669-78. 
39. Hiippala K, Kainulainen V, Kalliomäki M, Arkkila P, Satokari R. Mucosal Prevalence and Interactions with the Epithelium Indicate Commensalism of Sutterella spp. Front Microbiol 2016;7:1706.

40. Shallis RM, Terry CM, Lim SH. Changes in intestinal microbiota and their effects on allogeneic stem cell transplantation. Am J Hematol 2018;93:122-8.

41. Arias CA, Murray BE. The rise of the Enterococcus: beyond vancomycin resistance. Nat Rev Microbiol 2012;10:266-78.

42. Phillips RS, Sung L, Ammann RA, Riley RD, Castagnola E, Haeusler GM, Klaassen R, Tissing WJE, Lehrnbecher T, Chisholm J, Hakim H, Ranasinghe N, et al. Predicting microbiologically defined infection in febrile neutropenic episodes in children: global individual participant data multivariable meta-analysis. Br J Cancer 2016;114:623-30.

43. van Vliet MJ, Tissing WJE, Dun CAJ, Meessen NEL, Kamps WA, de Bont ESJM, Harmsen HJM. Chemotherapy Treatment in Pediatric Patients with Acute Myeloid Leukemia Receiving Antimicrobial Prophylaxis Leads to a Relative Increase of Colonization with Potentially Pathogenic Bacteria in the Gut. Clin Infect Dis 2009;49:262-70.

44. Steck N, Hoffmann M, Sava IG, Kim SC, Hahne H, Tonkonogy SL, Mair K, Krueger D, Pruteanu M, Shanahan F, Vogelmann R, Schemann M, et al. Enterococcus faecalis Metalloprotease Compromises Epithelial Barrier and Contributes to Intestinal Inflammation. Gastroenterology 2011;141:959-71.

45. Kim SO, Sheikh HI, Ha S-D, Martins A, Reid G. G-CSF-mediated inhibition of JNK is a key 
mechanism for Lactobacillus rhamnosus-induced suppression of TNF production in macrophages. Cell Microbiol 2006;8:1958-71.

46. Fite A, Macfarlane S, Furrie E, Bahrami B, Cummings JH, Steinke DT, Macfarlane GT. Longitudinal Analyses of Gut Mucosal Microbiotas in Ulcerative Colitis in Relation to Patient Age and Disease Severity and Duration. J Clin Microbiol 2013;51:849-56.

47. Nycz BT, Dominguez SR, Friedman D, Hilden JM, Ir D, Robertson CE, Frank DN. Evaluation of bloodstream infections, Clostridium difficile infections, and gut microbiota in pediatric oncology patients. PLoS One 2018;13:e0191232.

48. Montassier E, Al-Ghalith GA, Ward T, Corvec S, Gastinne T, Potel G, Moreau P, de la Cochetiere MF, Batard E, Knights D. Pretreatment gut microbiome predicts chemotherapyrelated bloodstream infection. Genome Med 2016;8:49.

49. Wardill HR, Tissing WJE. Determining risk of severe gastrointestinal toxicity based on pretreatment gut microbial community in patients receiving cancer treatment: A new predictive strategy in the quest for personalized cancer medicine. Curr Opin Support Palliat Care 2017;11:125-32.

50. Ciorba MA, Hallemeier CL, Stenson WF, Parikh PJ. Probiotics to prevent gastrointestinal toxicity from cancer therapy: an interpretive review and call to action. Curr Opin Support Palliat Care 2015;9:157-62. 
Table 1. Patients and disease characteristics $(\mathrm{N}=51)$

\begin{tabular}{|c|c|c|}
\hline & & Value \\
\hline \multirow[t]{2}{*}{ Sex, N (\%) } & Female & $13(25)$ \\
\hline & Male & $38(75)$ \\
\hline Age, median years (IQR) & & $3.7(2.7-6.8)$ \\
\hline \multirow[t]{3}{*}{ ALL phenotype, N (\%) } & pre-B-cell ALL & $43(84)$ \\
\hline & T-cell ALL & $7(14)$ \\
\hline & Other $^{1}$ & $1(2)$ \\
\hline \multirow[t]{3}{*}{ Induction, N (\%) } & Non-HR & $42(82)$ \\
\hline & HR & $5(10)$ \\
\hline & Other $^{2}$ & $4(8)$ \\
\hline \multirow[t]{4}{*}{ Final risk group, $\mathrm{N}(\%)$} & SR & $24(47)$ \\
\hline & IR & $20(39)$ \\
\hline & HR & $5(10)$ \\
\hline & Other ${ }^{3}$ & $2(4)$ \\
\hline \multirow[t]{3}{*}{ Treating center } & $\mathrm{OUH}$ & $15(29)$ \\
\hline & $\mathrm{RH}$ & $33(65)$ \\
\hline & AUH & $3(6)$ \\
\hline
\end{tabular}

ALL, acute lymphoblastic leukemia; SR, standard risk; IR, intermediate risk; HR, high risk; OUH, Odense university hospital; RH, Copenhagen university hospital; AUH, Aarhus university hospital. ${ }^{1}$ Blastic plasmacytoid dendritic cell acute leukemia treated with NOPHO ALL2008 protocol; ${ }^{2}$ Two patients start HR-block therapy before the end of induction treatment and two Ph+ ALL patients receive non-HR induction treatment with Imatinib from day $15 ;{ }^{3}$ Two patients with $\mathrm{Ph}+\mathrm{ALL}$. 
Table 2. Associations between selected bacterial species and plasma CRP and citrulline levels on each day by Spearman's rank correlations

\begin{tabular}{|c|c|c|c|c|}
\hline Plasma parameters & Species (abundance) & Day & rho & p-value ${ }^{1}$ \\
\hline \multirow[t]{8}{*}{ CRP (mg/L) } & Unclassified Enterococcus spp. & 8 & 0.28 & 0.061 \\
\hline & & 15 & 0.10 & 0.598 \\
\hline & & 22 & 0.42 & 0.009 \\
\hline & & 29 & 0.49 & 0.001 \\
\hline & Sutterella wadsworthensis & 8 & -0.07 & 0.641 \\
\hline & & 15 & -0.33 & 0.064 \\
\hline & & 22 & -0.24 & 0.154 \\
\hline & & 29 & -0.35 & 0.031 \\
\hline \multirow[t]{4}{*}{ Citrulline $(\mu \mathrm{M})$} & Unclassified Lachnospiraceae spp. & 8 & 0.48 & 0.001 \\
\hline & & 15 & 0.62 & $<0.001$ \\
\hline & & 22 & 0.24 & 0.152 \\
\hline & & 29 & 0.18 & 0.286 \\
\hline
\end{tabular}


Figure legends:

Figure 1. Clinical intestinal mucositis (IM) severity (A), plasma citrulline (B) and plasma CRP

(C) levels during induction treatment. Asterisks indicate statistically significant differences compared to baseline ( $* \mathrm{P}<0.05, * * \mathrm{P}<0.01, * * * \mathrm{P}<0.001)$. Boxes represent the median and interquartile range. The dashed horizontal line indicates the normal CRP threshold (10 mg/L).

Figure 2. Number of observed species (A) and Shannon index (B) during induction treatment. Asterisks indicate statistically significant differences compared to day $1\left({ }^{*} \mathrm{P}<0.05,{ }^{*} \mathrm{P}<0.01\right.$, $* * * \mathrm{P}<0.001)$. Boxes represent the median and interquartile range.

Figure 3. Associations between same-day Shannon diversity index and plasma CRP (A) and citrulline (B) levels. (A) Spearman’s rank correlations coefficients (r) between Shannon index and plasma CRP on day $15(\mathrm{P}=0.023)$, day $22(\mathrm{P}=0.002)$ and day $29(\mathrm{P}=0.042)$. (B) Spearman's rank correlations coefficients $(r)$ between Shannon index and plasma citrulline on day $15(\mathrm{P}=0.058)$ and day $29(\mathrm{P}=0.050)$.

Figure 4. Relative abundance of unclassified Enterococcus spp. (A) and Lachnospiraceae spp.

(B) during induction treatment. Asterisks indicate statistically significant differences compared to 
baseline $(* \mathrm{P}<0.05, * * \mathrm{P}<0.01)$. Boxes represent the median and interquartile range. $\mathrm{Y}$-axes are logtransformed. 

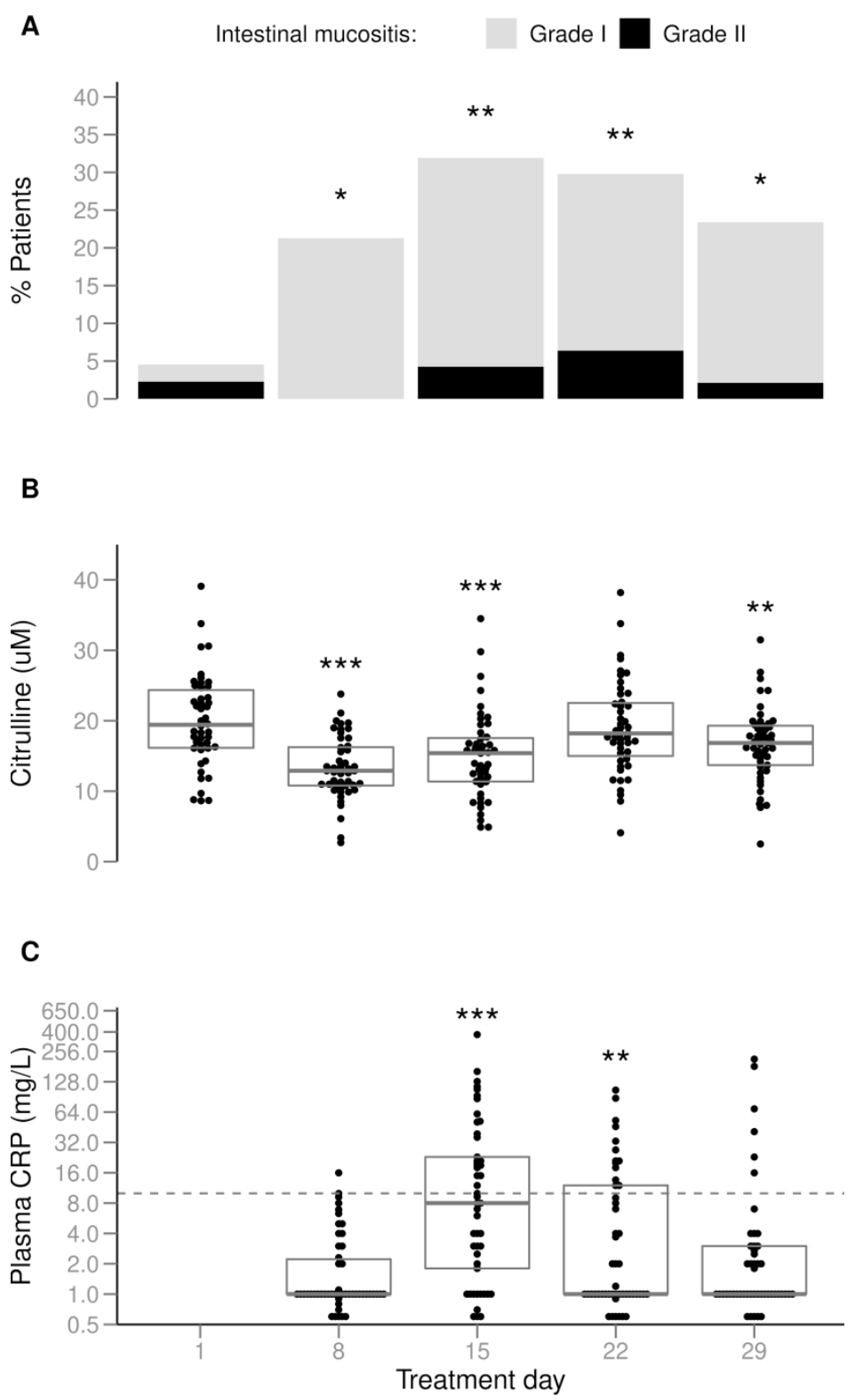

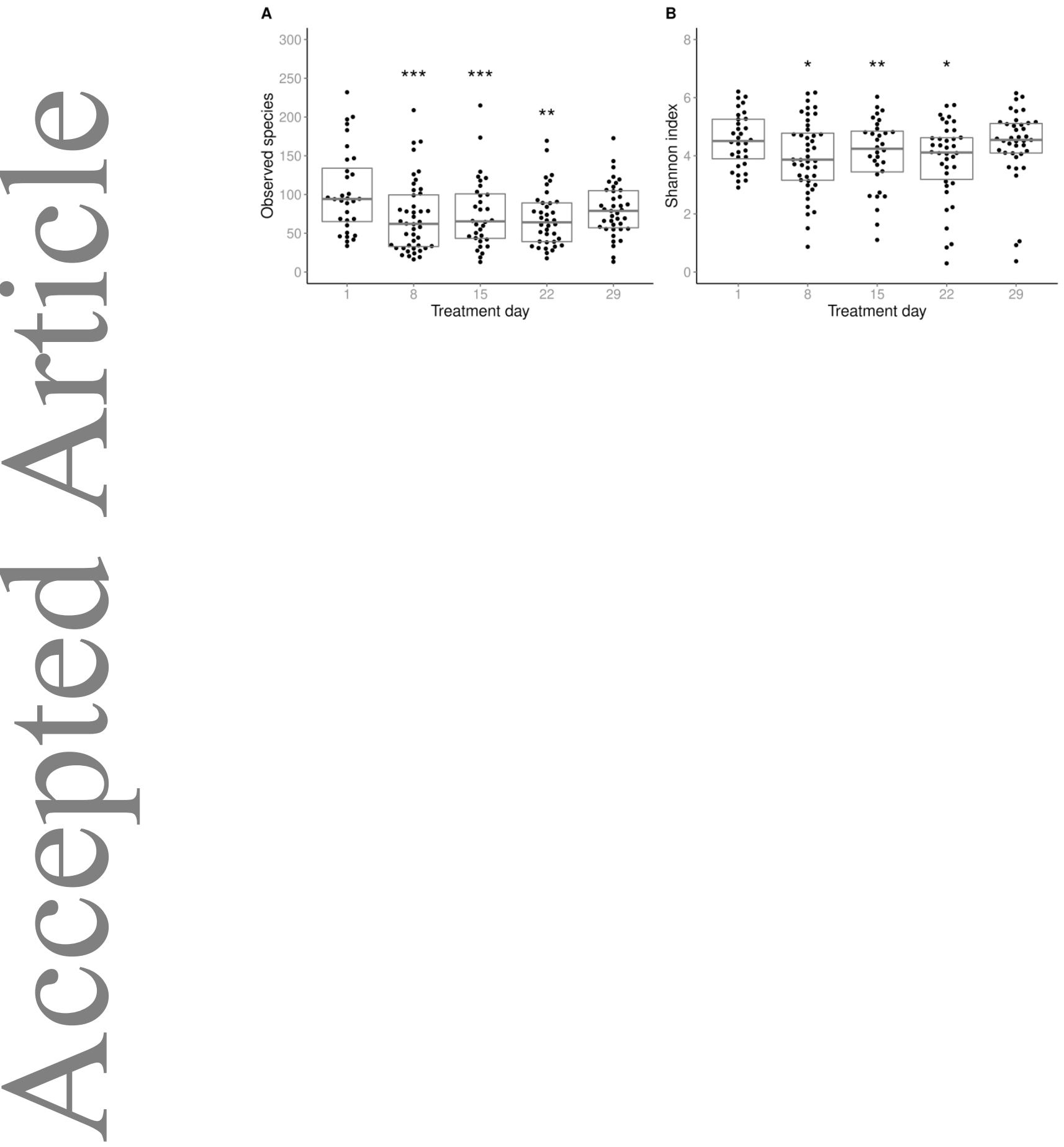

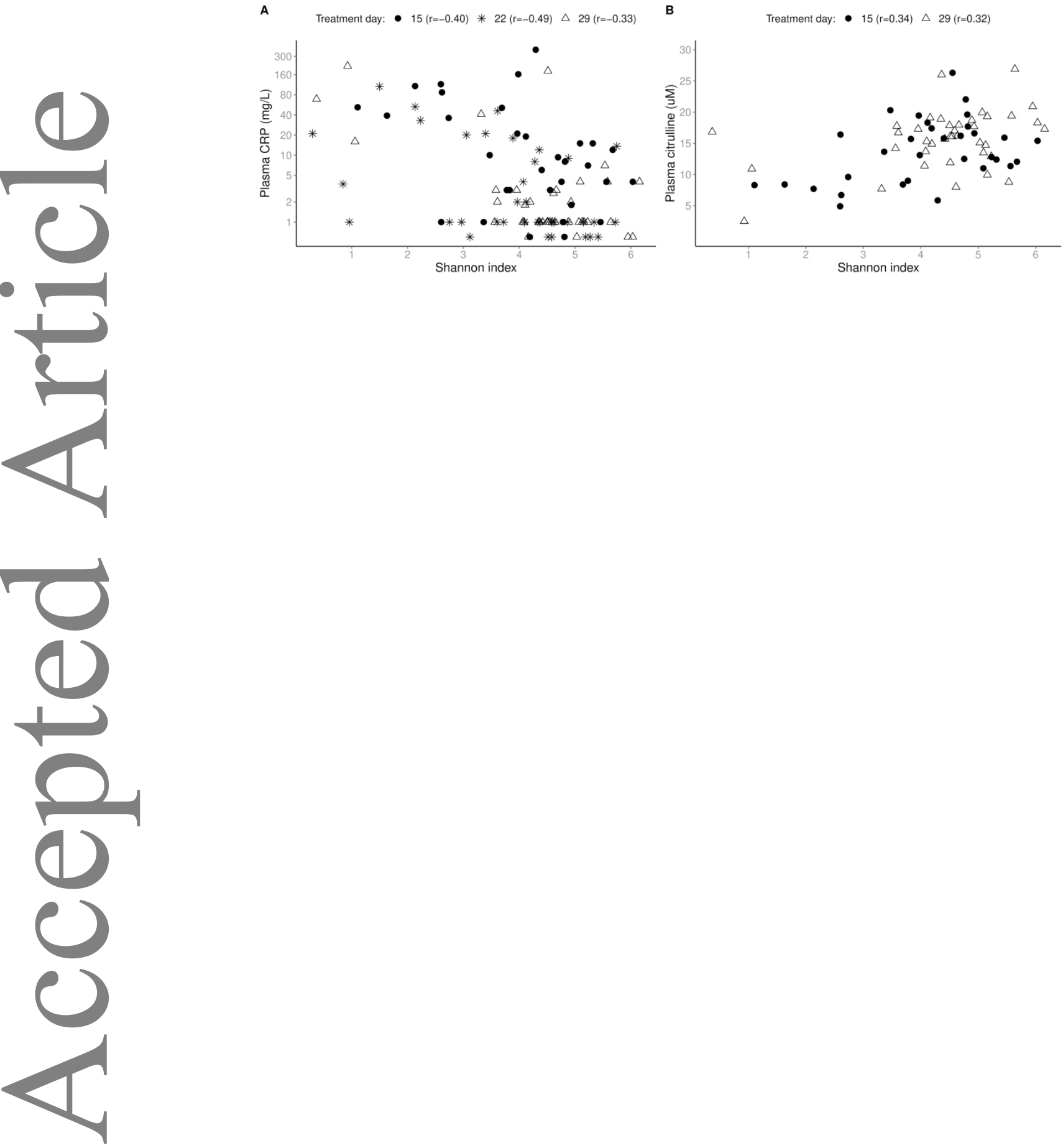

This article is protected by copyright. All rights reserved. 


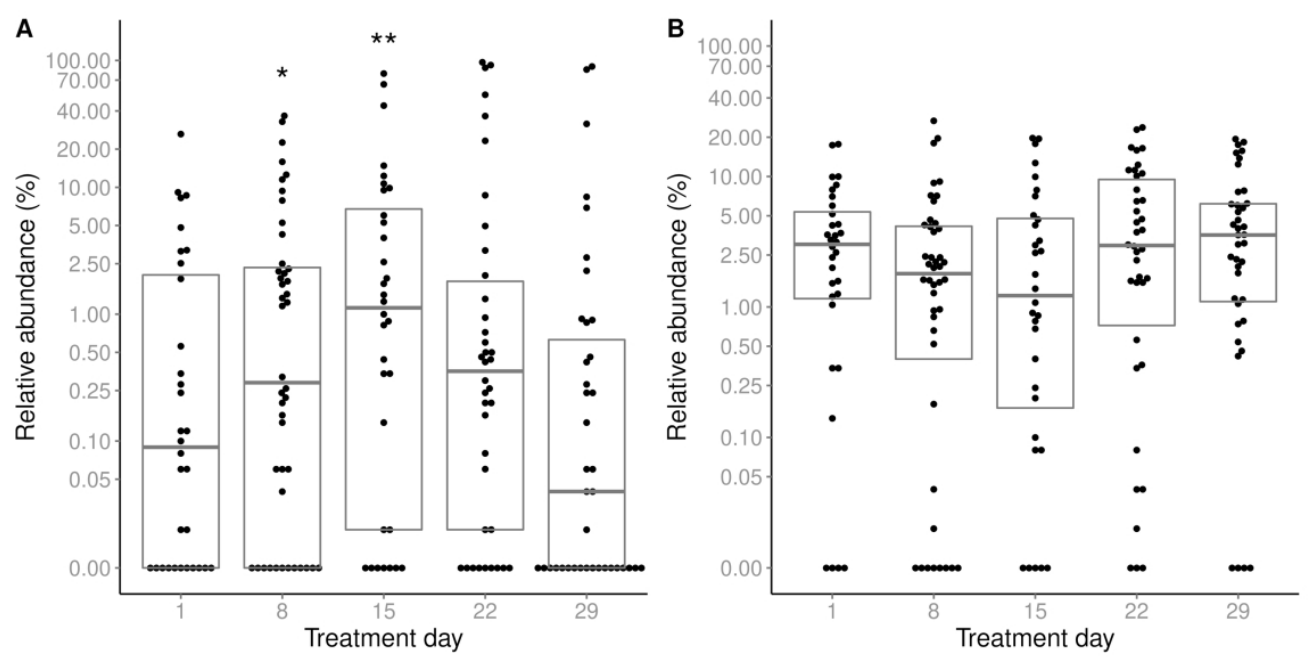


neser

Applications in CAR T-cell Therapy: Dissecting Cellular Composition Using Single Cell Multiomics

Tuesday, March 24th (US)

5:00pm EST, 2:00pm PST

Wednesday, March 25th (AU)

8:00am NSW
Free Webinar Register Now

Sponsored by

Provided by

BD ECURRENT WILCOL WILEY 